\title{
9 A TERAPIA COMUNITÁRIA - CRIANDO REDES SOLIDÁRIAS EM UM CENTRO DE SAÚDE DA FAMÍLIA
}

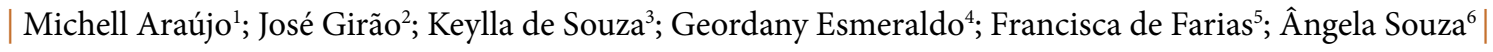

\section{RESUMO}

A terapia comunitária constitui uma estratégia do enfermeiro na atenção primária em saúde, pois acolhe a demanda do serviço, direciona os casos mais graves e possibilita integração comunitária. O objetivo deste artigo é relatar a experiência da Terapia Comunitária como estratégia de promoção da saúde mental em um Centro de Saúde da Família na cidade de Fortaleza-Ceará-Brasil. Ao realizarmos a terapia não trabalhamos simplesmente os sintomas, mas especialmente os sentimentos, os desejos e as necessidades das pessoas, elas são convidados a mergulharem em suas raízes culturais, religiosas e comunitárias, aprendem a partilhar com outros, suas dores e histórias de vida, constroem seus próprios caminhos a partir de si mesmas e conseguem transformar seu sofrimento em crescimento pessoal. Percebemos que a Terapia Comunitária mostra-se uma eficaz estratégia de primeira escuta comunitária, e vemos claramente sua importância como um trabalho viável que cuida não somente de pessoas doentes, mas especialmente promove a saúde mental.

PALAVRAS-CHAVE: Promoção da saúde; Saúde mental; Saúde da família

\section{RESUMEN}

"La Terapia Comunitaria - creación de redes de solidaridad en un Centro de Salud Familiar"

La terapia comunitaria es una estrategia de la enfermería en la atención primaria de la salud, que da la bienvenida a la demanda del servicio, dirige los casos más graves y permite la integración comunitaria.El objetivo de este trabajo es reportar la experiencia de la terapia comunitaria como una estratégia para promover la salud mental en un Centro de Salud de la Familia de Fortaleza-CearáBrasil. Al realizarmos la terapia no trabajamos sólo los síntomas, pero sobre todo los sentimientos, los deseos y necesidades de las personas, se les invita a sumergirse en sus comunidades culturales, religiosas y aprender a compartir con los demás sus tristezas y sus historias de vida, construir sus propios caminos desde sí mismos y transformar su sufrimiento en el crecimiento personal. Nos dimos cuenta de que la terapia resulta em una estrategia eficaz de primera escucha comunitaria, y vemos claramente su importancia como una tarea factible que no sólo se ocupa de los enfermos, pero sobre todo promueve la salud mental.

\section{DESCRIPTORES: Promoción de la salud; Salud mental; Salud} de la familia

\begin{abstract}
"The Community Therapy - creating networks solidarity in a Family Health Center"

Community therapy is a nursing strategy in primary health care, it welcomes the demand of the service, directs the most serious cases and enables community integration. The objective of this article is to tell the experience of Community Therapy as strategy of mental health promotion in a Family Health Center of FortalezaCeará-Brazil. When accomplishing therapy we don't work only symptoms, but especially feelings, desires and people's needs, they are invited to go deep in their cultural, religious and community bases, learning how to share with others their pain and history of life, they build their own ways from themselves and are able to transform their suffering in personal growth. We noticed that Community Therapy is an effective strategy of first community listening and we clearly see its importance as a viable work that takes care not only of sick people but especially promotes mental health.
\end{abstract}

\section{KEYWORDS: Health promotion; Mental health; Family health}

Submetido em 30-03-2016

Aceite em 05-01-2018

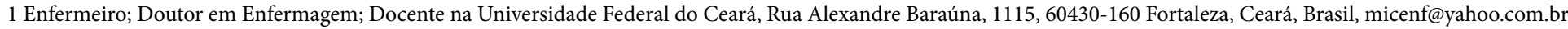
2 Enfermeiro; Bacharel em Enfermagem, evangleyson_01@yahoo.com.br

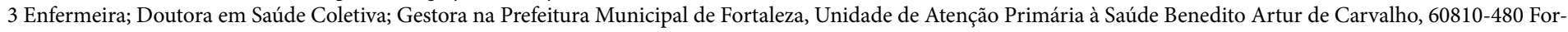
taleza, Ceará, Brasil, keyllanurse@yahoo.com.br

4 Enfermeira; Mestre em Saúde Pública; Docente na Universidade de Fortaleza, 60811-905 Fortaleza, Ceará, Brasil, geordanyrose@yahoo.com.br

5 Enfermeira; Doutora em Enfermagem Psiquiátrica; Docente na Universidade de Fortaleza, 60811-905 Fortaleza, Ceará, Brasil, luceliafarias@unifor.br

6 Enfermeira; Doutora em Enfermagem; Docente na Universidade Federal do Ceará, 60430-160 Fortaleza, Ceará, Brasil, amasplus@yahoo.com.br
} 


\section{INTRODUÇÃO}

Segundo a Organização Mundial da Saúde (OMS) $20 \%$ da população mundial sofre de algum transtorno mental no mundo. No Brasil esse número é um pouco menor, mas não menos preocupante. De acordo com o Ministério da Saúde $12 \%$ da população brasileira passa por algum sofrimento psíquico. Esse número é alarmante quando pensamos na necessidade de apoio e tratamento para as pessoas com algum sofrimento, trazendo repercussões para a gestão da saúde e principalmente para os serviços que recebem a demanda cada dia maior (Mielke \& Olschowsky, 2011).

A elaboração de estratégias faz-se mister, a fim de assegurar uma atenção de qualidade, respeitando a dignidade e a cidadania dos indivíduos conforme recomenda a Reforma Psiquiátrica Brasileira. Nas discussões sobre as novos modos de fazer saúde no Brasil a partir dos movimentos de redemocratização, das conferências de saúde, sobretudo a VIII Conferência e a criação do Sistema Único de Saúde, a saúde mental passou a ser pauta importante nas mudanças necessárias, do modelo manicomial para um modelo comunitário e de reabilitação psicossocial (Amarante, Lepre, Gomes, Pereira, e Dutra, 2011).

A atenção primária tem papel importante na assistência psiquiátrica e de saúde mental. Desde a Conferência de Alma Ata em 1978, esse nível de atenção tem sido evidenciado como fundamental à promoção da saúde, principalmente na colaboração da reorganização do setor (Kemppainen et al., 2012).

A Conferência de Promoção da Saúde realizada em Ottawa em 1986 que estabelece a direção das novas políticas de saúde reafirmam a justiça social, a equidade, a defesa da saúde e a mediação política para alcançá-la. Em um dos cinco campos de atuação para a promoção da saúde identificados em Ottawa encontra-se a reorganização dos serviços de saúde. Assim como em Alma Ata, essa conferência dispensa especial atenção ao nível primário de saúde para assegurar e organizar os serviços básicos e indispensáveis a população e de toda a rede inclusive os níveis de maior complexidade (Kemppainen et al., 2012).

Poderíamos nos perguntar: por que privilegiar o nível primário em saúde? É nesse nível que se encontra a maior possibilidade de atuação e aproximação da família e da comunidade, favorecendo assim o manejo de casos mais simples, criando uma porta de entrada para serviços especializados e favorecimento do apoio, da socialização comunitária e da participação popular (Mielke \& Olschowsky, 2011).
Como enfermeiros e profissionais de saúde, observando a demanda e os principais problemas de saúde das pessoas atendidas no centro de saúde da família que atuamos, notamos que há um alto índice de uso de benzodiazepínicos, casos de depressão, tentativas de suicídio e muitos casos de fobias, síndrome do pânico e dependência química, além de dificuldades familiares, financeiras, ambientais e religiosas que envolvem não somente a família, mas a comunidade de modo geral. Muitos questionamentos surgiram a partir dos problemas detectados: Como atender essa demanda? Encaminhar aos serviços de saúde mental? Trazer profissionais de saúde mental para atender no centro de saúde? Seríamos capazes de prestar assistência a essas pessoas? Como faríamos? Pensamos em muitas possibilidades de atendimento dessa demanda no próprio centro de saúde da família. Uma das estratégias que resolvemos implementar foi a Terapia Comunitária (TC), por tratar-se de uma tecnologia leve de cuidado em saúde, por isso não teria muitos custos ou dificuldades (Padilha, Oliveira, 2012).

Essa estratégia foi criada pelo psiquiatra Adalberto de Paula Barreto, professor da Universidade Federal do Ceará, ao encontrar no bairro Pirambú, na periferia de Fortaleza, uma demanda humanamente impossível para um único médico realizar atendimento. Ele começou a fazer grupos e tentar praticar a escuta terapêutica, descobriu que o próprio grupo é sábio e pode encontrar suas respostas nele mesmo. Percebeu que sua atuação deveria ser cada vez menos intervencionista, encontrou assim um modo de potencializar a auto-estima dessa comunidade e despertá-la para a busca, a partir de si mesma, de soluções para os problemas (Barreto, 2011). Tem como pilares: o pensamento sistêmico, a antropologia cultural, a teoria da comunicação, a pedagogia de Paulo Freire e a resiliência. A contribuição da TC na promoção da qualidade de vida das pessoas tem chamado a atenção de autoridades governamentais. Estes benefícios fundamentaram o posicionamento dessa estratégia como uma política pública em várias áreas como a educação, saúde, segurança pública, justiça e ação social. O baixo custo, a alta efetividade, o empoderamento das comunidades e a partilha de soluções participativas alicerçam a TC como uma política pública adequada no atendimento das diversas e complexas demandas (Baratieri, 2013; Barreto, 2011).

Outro dado relevante que favoreceu o apoio governamental diz respeito à grande capilaridade do movimento da TC no Brasil. 
A rede é ampla, contando com 30.000 terapeutas comunitários que atuam em suas comunidades como voluntários ou associando a TC à sua atuação profissional pública ou privada (Lazarte, 2015). A formação e o acompanhamento dos grupos também encontram-se estruturados. Todas as regiões brasileiras possuem pólos de formação, num total de 36 , e os terapeutas comunitários estão organizados pela Associação Brasileira de Terapia Comunitária. A TC ainda conta com uma experiência exitosa fora do país, como no caso da França e dos EUA (Carvalho, Romero, e Ferreira Filha, 2013; Barreto, 2011; Carvalho et al., 2013).

A sessão é conduzida por um terapeuta comunitário e um co-terapeuta que sempre começam com acolhimento onde as pessoas são apresentadas, utilizando para isso músicas, dinâmicas e vivências. Relatam-se as regras: respeito quando o outro falar; evitar os conselhos e julgamentos; utilizar somente a primeira pessoa do singular em sua fala; e valorizar o conteúdo da partilha dos outros participantes, entre outras. Em seguida as pessoas são convidadas a partilhar com o grupo seus sofrimentos, problemas e dificuldades, cada participante tem espaço para falar e ser escutado. Dentre as histórias relatadas apenas uma é escolhida e discutida naquela sessão. Feita a escolha da história, as pessoas podem fazer perguntas esclarecedoras, nunca curiosas, sobre a história em discussão. Aprofundado o problema, o terapeuta lança o mote, que é a pergunta que envolve a todos, um exemplo é o mote simples: "quem aqui já passou por isso e o que fez para resolver?". É nesse momento onde ocorrem as trocas de experiências e modos de resoluções dos problemas vivenciados. Finaliza-se a terapia com a conotação positiva (valorização dos participantes), agradecimentos e breve avaliação do grupo (Barreto, 2011).

A TC surge como estratégia de primeira escuta comunitária, o terapeuta não necessariamente é um profissional de nível superior ou integrante da equipe de saúde da família, podem ser líderes comunitários ou pessoas da comunidade devidamente treinadas e formadas para atuarem como terapeutas. Esse fato possibilita o envolvimento das pessoas da própria comunidade na rede de saúde e os torna co-responsáveis nas decisões e resoluções dos problemas locais.

Vemos claramente a importância de utilizarmos estratégias viáveis na saúde mental, para não somente cuidarmos de pessoas doentes, mas especialmente na promoção de saúde, nisso a terapia comunitária constitui estratégia ímpar à atenção primária e para o programa de saúde da família.
O objetivo desse artigo é relatar a nossa experiência ao utilizar a terapia comunitária como estratégia de promoção da saúde mental na atenção primária em um centro de saúde da família da cidade de Fortaleza, Ceará, Brasil.

\section{MÉTODOS}

Trata-se de um estudo descritivo, tipo relato de experiência. Organizamos para descrever o relato a seguinte sequência de apresentação: o cenário, os participantes, a terapia, a avaliação das sessões. Os resultados são descritos e discutidos para evidenciar os efeitos da Terapia Comunitária nos participantes e sua importância para os enfermeiros de saúde mental (Brito et al., 2013; Sobrinho et al., 2011)

O Cenário: $\mathrm{O}$ relato da experiência aconteceu no espaço do Centro de Saúde da Família Benedito Artur de Carvalho (CSF-BAC), situado no município de Fortaleza-CE. O CSF-BAC conta com quatro equipes de Saúde da Família, além de ginecologia, pediatria, clínica geral, psicologia, nutrição, enfermagem, odontologia e profissionais de nível médio. A população coberta pela unidade é de 25.107 habitantes (Secretaria Municipal de Saúde de Fortaleza, 2015).

O Sistema Municipal de Saúde de Fortaleza em 2015, contava com 248 equipes de Saúde da Família distribuídas em 86 Centros de Saúde da Família. Apresentava capacidade instalada para a realização de serviços primário, secundário e terciário. Integra a rede regionalizada e hierarquizada do SUS, sendo referência em âmbito micro, macrorregional e interestadual. A gestão do Sistema, em âmbito local, é de responsabilidade da Secretaria Municipal de Saúde (Secretaria Municipal de Saúde de Fortaleza, 2015).

Os Participantes: as pessoas foram encaminhadas por meio de consultas médicas, de enfermagem e odontológicas, dos agentes comunitários de saúde, do serviço de psicologia, do acolhimento do centro de saúde, da demanda espontânea, ou mesmo trazidas pelas pessoas que já participaram da terapia, além de diversos serviços de saúde da Regional II.

As pessoas que freqüentavam eram em sua maioria mulheres que apresentam ansiedades leves, depressão e problema gerais como: baixa auto-estima; problemas familiares; sintomas psicossomáticos; inadequação social; dificuldade financeira, dependência química ou de algum familiar; distúrbios mentais graves; adaptação ao climatério e/ou ao envelhecimento; idéias ou tentativa de suicídio. 
Não havia nenhuma avaliação prévia, qualquer pessoa que comparecer a sessão é acolhida e incentivada a participar.

Somente depois da sessão, no momento da avaliação entre o terapeuta e o co-terapeuta, alguns encaminhamentos foram realizados, principalmente ao se tratar de pessoas com doença mental grave ou que tinham uma demanda para psicoterapia individual.

A Terapia: todo o processo era registrado em um diário de campo desde o planejamento das sessões, as sessões em si e suas avaliações.

Ocorriam semanalmente, às terças-feiras às $18 \mathrm{~h}$ e tinha duração de duas horas. Em média participam 12 pessoas, era um grupo aberto, podendo cada sessão ter pacientes novos e veteranos, não se determinava limite máximo de participantes a não ser pelo espaço restrito na unidade de saúde, eram em sua maioria adultos e alguns idosos. Já passaram pela terapia segundo nosso registro mais de 127 pessoas desde o inicio das sessões no final de 2007 até 2015.

Os pacientes apresentavam em sua maioria queixas difusas, não havia diagnósticos médicos estabelecidos em todos, acorriam para o grupo pessoas ansiosas, deprimidas, com doenças crônicas e que apresentavam sofrimento de âmbito pessoal, familiar, laboral e socioeconômico. Eram encaminhados pelas equipes de saúde da família ou participavam espontaneamente aqueles que sabiam que havia terapia no centro de saúde. Nas sessões havia sempre a presença de um terapeuta e um co-terapeuta, mas contávamos com uma equipe de quatro terapeutas, que se revezavam, conforme escala estabelecida pela equipe de terapeutas, no caso eram enfermeiros das equipes de saúde da família com formação em terapia comunitária.

A Avaliação: a cada sessão realizávamos duas avaliações, no final de cada sessão com os participantes e após finalizarmos a terapia uma avaliação era realizada entre os terapeutas.

Utilizávamos três perguntas simples com os participantes com os terapeutas: $O$ que houve de bom na sessão? O que não deveria ter acontecido na sessão? E como melhorar as próximas sessões?

Mesmo tratando-se de um relato de experiência todos os princípios éticos forma seguidos, conforme as recomendações nacionais e internacionais de pesquisa. Obtivemos a anuência da instituição pesquisada para realizar a experiência e relatá-la posteriormente.

\section{RESULTADOS E DISCUSSÃO}

Os efeitos sobre as pessoas que participaram da terapia: Muitas vezes chegavam completamente comprometidas, sofridas e com vidas interrompidas devido aos sintomas. Muitas pessoas não conseguiam mais trabalhar, estudar e realizar suas atividades cotidianas, principalmente pelos sintomas como: medo, insônia, dor no peito, palpitação, falta de ar e tremores.

$\mathrm{Na}$ terapia não trabalhávamos simplesmente os sintomas, mas especialmente os sentimentos, os desejos e as necessidades. Solicitávamos que se desprendessem dos sintomas e começassem a olhar pra si mesmas. Na TC havia espaço propício à escuta, pessoas que nunca se permitiram falar foram incentivadas a abrirem à boca, mesmo que considerassem o conteúdo sem importância, ao participarem sentiam-se valorizadas e respeitadas.

De modo simples foi explicado o processo saúde/doença mental, foram lembradas com os ditados populares que esse processo acontece durante a vida e muitos dos sintomas surgem em decorrência de problemas não enfrentados ou negados, exemplos desses ditados são: quando a boca cala o corpo fala e quem guarda azeda, quando azeda estraga, quando estraga estoura, quando estoura fede. As pessoas foram convidadas a quebrar esse processo de adoecimento com sua partilha, ao deixar a boca falar, o corpo tende a calar e, além disso, foram admoestadas a não guardar nada, tentar resolver seus problemas no aqui e agora, não reservando espaço para a mágoa ou ressentimentos (Barreto, 2011).

Nos encontros as pessoas têm permissão de serem elas mesmas, toda a sessão foi intercalada de expressões espontâneas, com o uso da música, da poesia, das piadas e dos ditados populares. O choro como modo de expressar os sentimentos reprimidos foi acolhido com belas cantigas de antigamente, entre elas: encosta tua cabecinha no meu ombro e chora e conta logo tuas mágoas todas para mim... e se algum dia na vida você de mim precisar, saiba que sou seu amigo pode comigo contar.... Ou ainda deixa a luz do céu entrar, abre bem as portas do teu coração e deixa a luz do céu entrar... Havia uma descoberta na terapia que foi proporcionada pelo processo grupal, descobre-se lá que eu não sou o único sofredor no mundo, há muitos outros que sofrem tanto quanto eu ou mesmo, o meu problema não chega perto em grau de dificuldade em comparação ao do outro (Movimento de Saúde Mental Comunitária do Bom Jardim, 2010). 
A valorização da história pessoal de cada participante foi ressaltada porque constitui ponto importante da terapia, toda a trajetória de vida foi profundamente explorada quando se solicita que relate quem enfrentou problema semelhante e o que fez para resolver. Foi salientada a força, as vitórias e os obstáculos transpostos, mesmos se forem pequenas coisas realizadas. Revisitando as histórias de vida dos participantes, podíamos descobrir coisas nunca antes analisadas ou percebidas, encontrar respostas e soluções dentro de nós mesmos, isso a terapia propiciou, pois fez circular as experiências acumuladas.

Outro efeito que é preciso ressaltar nos participantes foi a rede solidária formada a cada sessão. Muitos encontros se desdobravam na mobilização de todos para ajudar a resolver determinados problemas. Em outros as resoluções e oportunidades surgiam no contato das pessoas entre si quando relatavam seus problemas para o grupo. Surgia a partir desse encontro um sentimento de pertença, que criava um ambiente acolhedor e convidativo para o próximo encontro. Os relacionamentos sociais que se estabelecem entre grupos terapêuticos propiciam o estar com o outro em diversas perspectivas e a relação com eles ocorre segundo variados níveis de proximidade, profundidade e anonimato na vivência (Nasi e Schneider, 2010).

A resiliência é algo que precisa ser destacado. Nesse princípio os participantes descobriam sua pérola. Fazendo analogia com a história da ostra que ao abrirse para se alimentar deixa entrar grãos de areia em seu interior que ferem seu corpo, mas ao ferir, a ostra produz uma substancia que envolve o grão dando origem à pérola, em outras palavras, é a capacidade de acabar com o sofrimento usando ele próprio e a partir dele buscar melhorar, a isso chamamos resiliência (Béné et al., 2014).

A resiliência revelada pela terapia nada mais é que a transformação do sofrimento em crescimento pessoal. Produzindo resposta a pergunta: O que você está fazendo com o seu sofrimento, crescendo ou sofrendo? Também presente em sua história de vida havia coisas importantes que o sofrimento pode trazer: aprendizado, maturidade, fortaleza, paciência e realizar uma transmutação do fraco em forte, do infantil em maduro, do desesperado em otimista.

Dentro dessa perspectiva, essa transformação realizada se reveste na descoberta das minhas competências, muitas delas provenientes dos sofrimentos vividos e por sua vez no fortalecimento da rede de apoio criada no grupo.
Víamos pessoas que já não mais precisavam das terapias, mas continuavam participando, sem terem consciência muitas vezes, que sua função era agora ajudar as pessoas que enfrentavam os mesmos problemas que haviam enfrentado anteriormente. Foram estimuladas a partilhar sua experiência de dor e os caminhos percorridos para a resolução. Esse ínterim surge mais um indagação: quem mais apropriado para entender um sofredor que outro sofredor? A resiliência nos ensina que o sofrimento pode gerar competência e a terapia comunitária teve o objetivo de mostrar esse processo e sua possibilidade na vida das pessoas.

Longe de fazermos apologia ao sofrimento, que se confunde com masoquismo, lembrávamos que os sofrimentos evitáveis precisavam ser resolvidos, mas as pessoas eram lembradas que as dificuldades da vida servem para aprendermos e nos tornarmos pessoas mais fortes, esses momentos difíceis nos instrumentalizam para enfrentarmos adequadamente, sem sucumbir ao desespero ou ao desenvolvimento de sintomas psíquicos ou psicossomáticos. Ao invés de tentarmos nos livrar dos sintomas ou sofrimentos, fomos incentivados na terapia a perguntar o que eles queriam nos comunicar, ao ouvirmos a voz proveniente do corpo, a ansiedade pode diminuir e assim contribuir com a resolução do problema.

\section{IMPLICAÇÕES PARA A PRÁTICA CLÍNICA}

Observamos que a presença da Terapia Comunitária no centro de saúde da família contribuiu para demonstrar a importância do enfermeiro em saúde mental e desmistificar suas ações na atenção básica, mesmo que ainda a grande demanda das sessões seja de pessoas classificadas socialmente como doentes mentais.

A Terapia Comunitária constituiu uma estratégia viável de promoção da saúde ao passo que reorganizamos o serviço de saúde, evitando assim encaminhamentos desnecessários dos casos simples aos centros de atenção psicossociais. Além do que disponibiliza um serviço de saúde mental na própria comunidade, servido de acolhimento e triagem da demanda.

Outro ganho que não poderíamos deixar de relatar foi o empoderamento que a TC proporcionou aos seus participantes, aprenderam no circular da partilha comunitária, que são importantes e sábios, ao passo que encontraram as saídas para suas dificuldades, favoreceram sua auto-estima e se tornaram transformadores da realidade. Descobriram e redescobriram suas potencialidades humanas, geradoras de saúde. 
Nos encontros as pessoas mergulhavam em suas raízes culturais, religiosas e comunitárias, aprendiam a partilhar com outros suas dores e histórias de vida, construíam seus próprios caminhos a partir delas próprias e conseguiam transformar seu sofrimento em crescimento pessoal.

\section{CONCLUSÃO}

A Terapia Comunitária criada a partir da necessidade de uma comunidade carente na periferia de Fortaleza tem seu foco principal na promoção da saúde, visto que leva o indivíduo a se tornar consciente do seu processo de saúde e de seus fatores de fortalecimento e desgaste. Nossa experiência não muito diferente da original, também se deu na periferia, em um espaço de pobreza, exclusão e abandono. A demanda de pessoas com sofrimento psíquico nessas condições é sempre crescente, a solução mais cômoda seria para as equipes de saúde da família encaminharem para serviços especializados mais próximos ou prescreverem ansiolíticos, como é feito muitas vezes nesses casos, isso somente aumentaria os problemas dos serviços de saúde e dos pacientes. Seguindo a política municipal de saúde oferecemos a nossa comunidade um espaço de promoção da saúde mental, onde são discutidos problemas pessoais e comunitários, em uma roda onde não existe a figura do doutor ou do salvador que cura e salva. As pessoas foram levadas a pensar as soluções a partir de si mesmas, não longe de seus problemas e de seus sofrimentos, mas imbricados neles, não de modo individualista e solitária, mas coletiva e solidária.

\section{REFERÊNCIAS BIBLIOGRÁFICAS}

Amarante, A. L., Lepre, A. S., Gomes, J. L. D., Pereira, A. V., \& Dutra, V. F. D. (2011). Nursing mental health care strategies in brazilian family health program. Revista Texto \& Contexto Enfermagem, 20(1), 85-93.

Baratieri, T. (2013). Evaluation of primary health care in Brazil: A literature review. Primary Health Care, 144(3), 2-5.

Barreto, A. P. (2011). Terapia comunitária passo a passo. Fortaleza: Grática LCR.

Béné, C., Newsham, A., Davies, M., Ulrichs, M., \& Godfrey-Wood, R. (2014). Review article: Resilience, Poverty and Development. International Development, 26(5), 598-623.
Brito, S. S., Nóbrega, R. V., Santos, S. R., Bezerra, E. P., Costa, K. N. F. M., \& Costa, M. M. L. (2013). Systematization of nursing assistance in primary care to hipertensive: Experience report. Revista de Enfermagem da UFPE on line,7(8), 5345-5350.

Carvalho, M. A. P., Dias, M. D., Miranda, F. A. N., \& Ferreira Filha, M. O. (2013). Contributions by integrative community therapy to users of Psychosocial Care Centers (CAPS) and family members: Thematic oral history. Caderno de Saúde Pública, 29(10), 2028-2038.

Carvalho, M. A. P., Romero, R. O. G., \& Ferreira Filha, M. O. (2013). Community Therapy in the Center of Psychosocial Support: Concepts of nursing students. Revista de Enfermagem UFPE on line, 7(5), 4389-4394.

Lazarte, R. (2015). Sociología y terapia comunitária integrativa. Revista Uruguaya de Enfermería, 7(1), 67-76. Kemppainen, V., Tossavainen, K., \& Turunen H. (2012) Nurses' roles in health promotion practice: An integrative review. Health Promotion International, 28(4), 490-501.

Mielke, F. B., \& Olschowsky, A. (2011). Actions of mental health in family health strategy and the health technologies. Escola Anna Nery, 15(4), 762-768

Movimento de Saúde Mental Comunitária do Bom Jardim. (2010). Cantos, Dinâmicas e Poesias. Fortaleza: Encaixe.

Nasi, C., \& Schneider, J. F. (2010). Social relationships in the quotidian of users of a psychosocial care center: A phenomenological study. Online Brazilian Journal of Nursing, 9(1).

Padilha, C. S., \& Oliveira, W. F. (2012). Community therapy: Practice reported by professionals within SUS in Santa Catarina, Brazil. Revista Interface, 16(43), 1069-1086.

Secretaria Municipal de Saúde de Fortaleza. (2015). Relatório de Gestão. Fortaleza: Secretaria Municipal de Saúde de Fortaleza.

Sobrinho, T. A. O., Medeiros, C. P. P., Maia, M. R., Reis, T. C., Miranda, L. P., \& Costa, P. F. (2011). Academic and multiprofessional Integration in the PET - Health Program: Experiences and Challenges. Revista da ABENO, 11(1), 39-42. 